\title{
Simulation Implementation and Application Research of High Speed Milling Regeneration Type Chatter Based on MATLAB
}

\author{
Yuan Lingling $^{a}$, Mei Wentao ${ }^{b}$, Zheng Yongfeng $^{c}$ \\ Tianjin Bohai Vocational Technical College, Tianjin, China

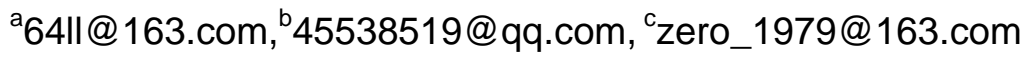

Keywords: Chatter stability domain; Machining process simulation; Milling

\begin{abstract}
Considering the deficiency in milling process parameters selection in the domestic. Based on the modeling of dynamic milling force and the calculate of chatter stability limits. Realizing high speed milling regeneration type chatter simulation algorithm with Matlab development tools. Through the modal hammer experiment, Obtaining the frequency response function to simulate the chatter stability domain graphics of the whole processing system which can be used as an instruction guide for the selection of milling process parameters. The validation and accuracy of the simulation algorithm was verified by experiments and used in a factory with an excellent application effect.
\end{abstract}

\section{Introduction}

At present, the hardware condition and production performance of high precision and high speed is provided by advanced high speed milling machining center of domestic and foreign in theory, but these devices failed to play maximum processing capacity, the reason is that the relevant problems of mechanical vibration in actual production Machining process. The production practices show that the slight chatter leads to the quality decline of the machined surface, reducing the production efficiency, Bearing overload and shortening the life of main shaft. Heavy chatter leads to serious breakage of the cutter and damage of work piece and spindle. Therefore, the research of causes, rules and suppression methods in high speed milling chatter can effectively avoid the instability caused by vibration ${ }^{[1-6]}$. this paper establishes regenerative type stability domain simulation flow chart of high speed milling and through the Matlab simulation algorithm for the analysis ${ }^{[7-9]}$. Selecting the stability parameters in order to avoid chatter , the simulation method has been applied in the factory and obtained the good application effect.

\section{Chatter Theory of High Speed Milling}

The Dynamic Model of Milling Force In High Speed Milling. The static milling force model without considering the effect of dynamic characteristic parameters in high speed milling, the cutting thickness of milling process was simply considered nonlinear relationship between feed rate and the instantaneous angle, ignoring the influence of vibration in milling process. Therefore, when the milling parameters change or workpiece vibration, the static milling force model can not descript about the true milling process, dynamics modeling has become an inevitable trend. The most critical for the dynamics modeling is the dynamic cutting thickness model, shown in Figure 1, expressed as:

$$
h_{D}\left(\phi_{j}\right)=f_{t} \sin \phi_{j}+\left(u_{j c}^{0}-u_{j c}\right)-\left(u_{j w}^{0}-u_{j w}\right)
$$

The milling dynamic system is simplified as Spring damping mass model in vibration mechanics, considering the influence of the previous cutter tooth left by corrugated surface, Instantaneous rigid force model is used in cutting tool, the influence of static deformation force is neglected. The Dynamic milling force is obtained by accumulating algorithm. In the time domain model, milling force model for differential forms expressed as follows: 


$$
\{F(t)\}=\frac{1}{2} a_{p} K_{t c}[A(t)]\{\Delta(t)\}
$$

In the formula $A(T)$ is a periodic function of $\omega=N \Omega, T=2 \pi / \omega$. This formula is decomposed according to Fourier series will simplify the calculation mode. Budak and Altintas[10, 11] have been proved the effect of periodic function harmonics on forecasting precision can be neglected in practical application. So after the time-varying system is transformed by Fourier series, only the first term is reserved. The formula is as follows:

$$
[A(0)]=\frac{1}{\phi_{p}} \int_{\phi_{s t}}^{\phi_{\phi_{x}}}[A(\phi)] d \phi=\frac{N}{2 \pi}\left[\begin{array}{ll}
a_{x x} & a_{x y} \\
a_{y x} & a_{y y}
\end{array}\right]
$$

The simplified milling force coefficient introduces into dynamic milling force formula, further simplified as:

$$
\{F(t)\}=\frac{1}{2} a_{p} K_{t}\left[A_{0}\right]\{\Delta(t)\}
$$

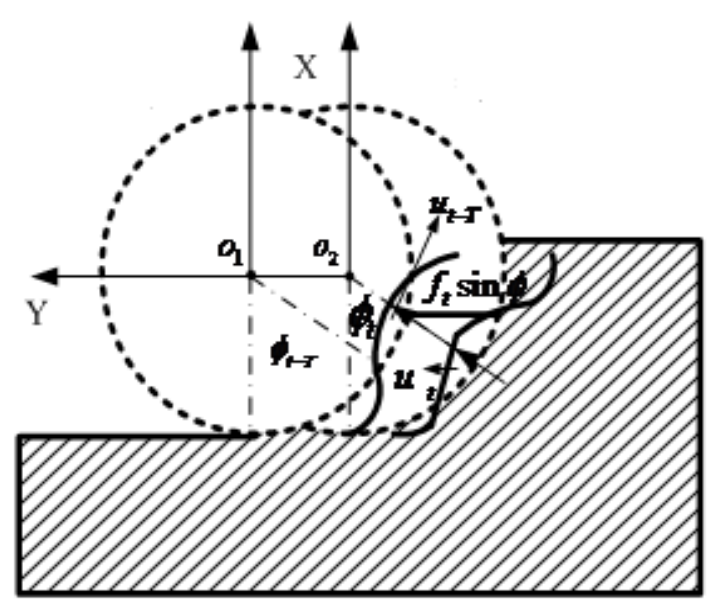

Fig. 1 Schematic diagram of the dynamic cutting thickness

The Chatter Stability Domain Algorithm. In the milling chatter stability analysis, Only considering the effects of dynamic milling force produced by the dynamic displacement of the tool and workpiece; The static part of the milling thickness is neglected.The main reason for the cutting depth and cutting direction changes is the periodic change of dynamic milling force coefficient matrix. Then the frequency response function of the tool and the workpiece is expressed as the following form:

$$
\begin{aligned}
& G_{x x}(s)=\frac{x(s)}{F_{x}(s)}=\frac{\omega_{n x}^{2}}{k_{x}\left(s^{2}+2 \zeta \omega_{n x} s+\omega_{n x}^{2}\right)} \\
& G_{y y}(s)=\frac{y(s)}{F_{y}(s)}=\frac{\omega_{n y}^{2}}{k_{y}\left(s^{2}+2 \zeta \omega_{n y} s+\omega_{n y}^{2}\right)}
\end{aligned}
$$

In the frequency domain analysis, the vibration vector equation described by harmonic function, and the equation is transformed into frequency domain equation, the transformation regeneration displacement equation as follows:

$$
\left\{\Delta\left(i \omega_{c}\right)\right\}=\left\{r\left(i \omega_{c}\right)\right\}-\left\{r_{0}\left(i \omega_{c}\right)\right\}=\left[1-e^{-i \omega_{c} T}\right] \cdot e^{i \omega_{c} t} \cdot\left[G\left(i \omega_{c}\right)\right] \cdot\{F\}
$$


The equation 4 is substituted into the frequency domain dynamic milling force model of 6 , Dynamic milling force considered the regenerative vibration is shown as follows:

$$
\{F\} e^{i \omega_{c} t}=\frac{1}{2} a_{p} K_{t c}\left(1-e^{i \omega_{c} T}\right)\left[A_{0}\right]\left[G\left(i \omega_{c}\right)\right]\{\Delta(t)\}\{F\} e^{i \omega_{c} t}
$$

In the chatter frequency, the mathematical formula is established on the flutter stability analysis of closed loop feedback dynamic milling system. also called the characteristic equation , the Related equation and solution As follows:

$$
\begin{aligned}
& \operatorname{det}[I]-\frac{1}{2} a_{p} K_{t c}\left(1-e^{i \omega_{c} T}\right) \frac{N}{2 \pi}\left[G_{0}\left(i \omega_{c}\right)\right]=0 \\
& \Lambda=-\frac{1}{2 a_{0}}\left(a_{1} \pm \sqrt{a_{1}^{2}-4 a_{0}}\right)
\end{aligned}
$$

\section{Among:}

$$
\begin{aligned}
& a_{0}=G_{x x}\left(i \omega_{c}\right) G_{y y}\left(i \omega_{c}\right)\left(\alpha_{x x} \alpha_{y y}-\alpha_{x y} \alpha_{y x}\right) \\
& a_{1}=\alpha_{x x} G_{x x}\left(i \omega_{c}\right)+\alpha_{y y} G_{y y}\left(i \omega_{c}\right)
\end{aligned}
$$

According to Euler's formula, considering the actual production and processing axial back engagement is real, Calculating system can steady cutting under the chatter frequency wc and the critical condition of the axial back engagement plural form is expressed as follows:

$$
a_{p \lim }=-\frac{2 \pi}{N K_{t c}}\left[\frac{\Lambda_{R}\left(1-\cos \omega_{c} T\right)+\Lambda_{I} \sin \omega_{c} T}{1-\cos \omega_{c} T}+i \frac{\Lambda_{I}\left(1-\cos \omega_{c} T\right)+\Lambda_{R} \sin \omega_{c} T}{1-\cos \omega_{c} T}\right]
$$

In the actual production of milling $a_{p}$ must be real, Therefore imaginary part of type 11 must be zero. Then simplified Limit formula of axial back engagement can be obtained as follows.

$$
a_{p \lim }=-\frac{2 \pi \Lambda_{R}\left[1+\kappa^{2}\right]}{N K_{t c}}
$$

Under the cutter tooth cutting cycle of $\mathrm{T}$ is known, We can solve the spindle speed relate to critical axial back engagement, expression is as follows:

$$
T=\frac{1}{\omega_{c}}(\xi+2 k \pi) \rightarrow n=\frac{60}{N T} \quad, \quad n=\frac{60}{N\left[(2 k+1) \pi-2 \tan ^{-1}\left(\Lambda_{I} / \Lambda_{R}\right)^{2}\right]}
$$

In the formula, K represents leaf number of the flutter stability analysis graphs.

After the above analysis, the ultimate expression of high speed milling chatter stability was obtained, Processing system dynamics parameters are obtained by using experimental modal analysis method (Hammering experiment), That is FRFs of the tip or the workpiece machining parts.

Simulation Program Design. Based on the above theoretical analysis, The simulation program was developed based on software MATLAB7.1, the simulation interface is designed based on MATLABGUIDE integrated environment (Graphical User Interface development environment) 、 ACCESS Database and Visio Basic. The simulation results are output in the form of graphic and data files. The program block diagram of the whole is shown in Figure 2, the simulation results of flutter stability region is shown in figure 3. With the similar foreign software such as CUTPRO contrast, Two simulation results are basically the same, but faster simulation speed. 


\section{Experimental verification}

In order to verify the validity and accuracy of chatter stability domain simulation algorithm, cutting experiment was carried out in the FIDIA D318 high speed machining center. The maximum speed of machine tool is $30000 \mathrm{r} / \mathrm{min}$. Tool used by the experiment is the whole hard alloy cutter of Sandvik, Parameters: diameter of $12 \mathrm{~mm}, 2$ teeth, 30 spiral angle, install overhang length is $70 \mathrm{~mm}$. The workpiece material is aluminum alloy AL12, specimen size is $100 \times 100 \times 20(\mathrm{~mm})$. Hammer test was conducted based on the "machine - tool" system. The X, Y direction of the FRFs is obtained. Compared with the tool, the box workpiece is considered a rigid body and it can be neglected when the simulation. Fig. 3 is the chatter stability lobes diagram of domain simulation. Validation experiments were performed in 6 selected points from the diagram (- said flutter, - said the stable cutting zones). Table 1 is the processing parameters used in the experiment and verification results. Fig. 3 and table 1 shows, flutter does not appear when the 1, 2, 3 points cutting parameters were used. While flutter appeared when the 4,5,6 points cutting parameters were used. This is fully consistent with the flutter stability domain simulation results. Therefore the flutter stability domain simulation algorithm is proved to be effective and accuracy.
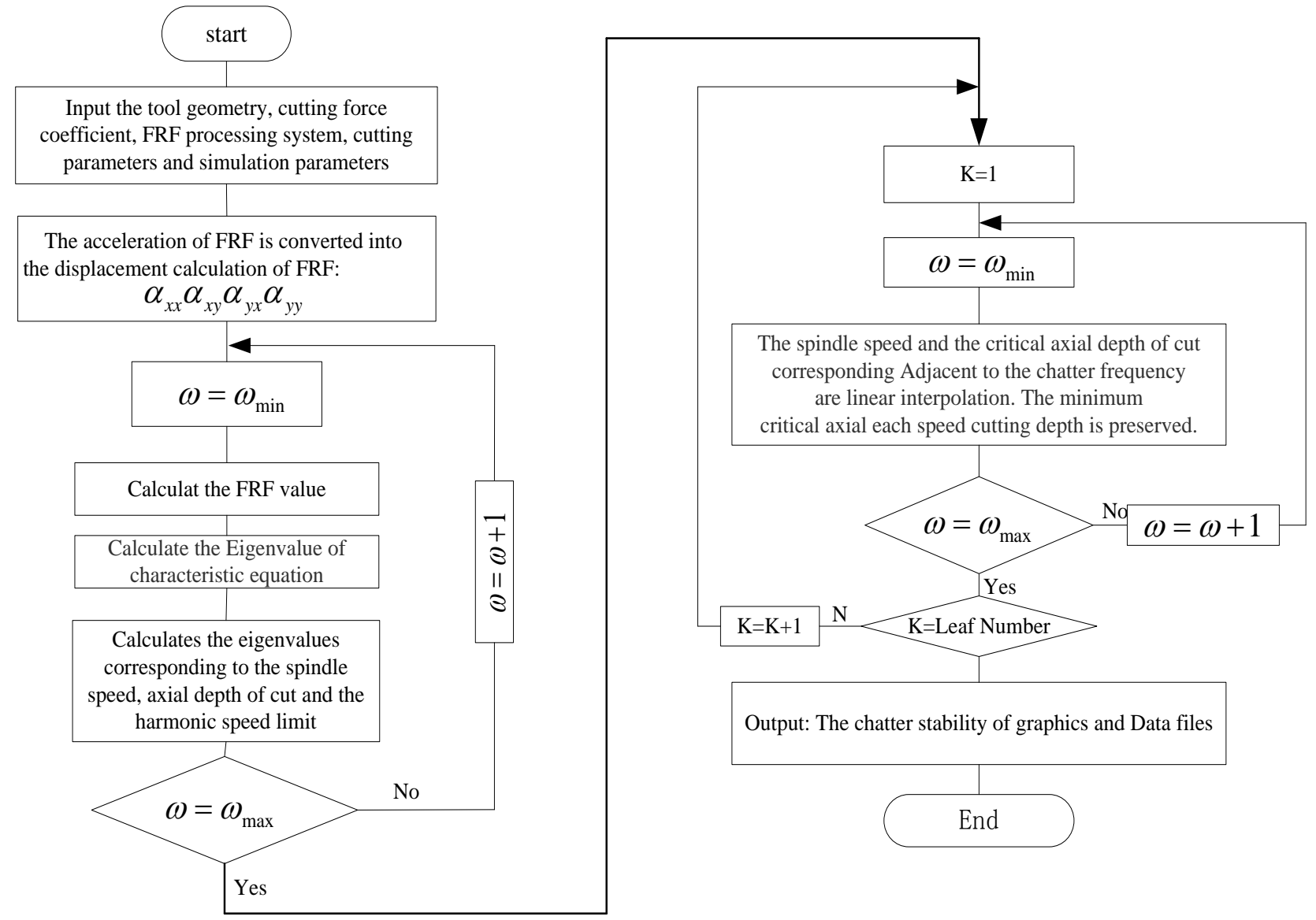

Fig. 2 The chatter stability lobes diagram 


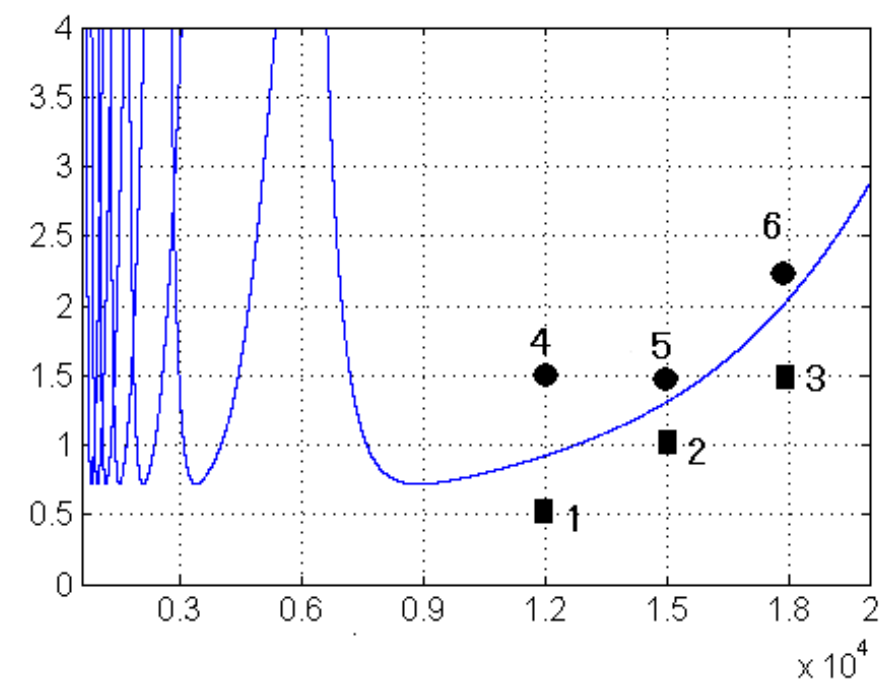

Fig.3 The simulation results

\section{Application analysis}

The front of the milling chatter stability domain simulation method has been applied and good results were obtained in a large state-owned enterprise. Now in the enterprise backbone machining center FIDIA D518 is taken as the example to illustrate. The maximum speed of FIDIA D518 is $40000 \mathrm{r} / \mathrm{min}$, the cutting parameters of previously used far less than this value (empirically selected). Dynamic parameter test and flutter stability domain simulation is carried on several tools, through the simulation experiment cutting parameters are optimized selection. The cutting parameters and the material removal rate comparison before and after optimization is shown in Table 2. Apparently, according to the simulation results of chatter stability lobes Cutting parameters are choose more reasonable, the processing efficiency is higher.

Table 1 The flutter stability domain verification (all slot milling, feed per tooth for $0.1 \mathrm{~mm}$ )

\begin{tabular}{c|c|c|c|c|c|c}
\hline Number & 1 & 4 & 2 & 5 & 3 & 6 \\
\hline Spindle Speed (r/min) & 12000 & 12000 & 15000 & 15000 & 18000 & 18000 \\
\hline The Critical cut depth (mm) & 0.5 & 1.5 & 1.0 & 1.5 & 1.5 & 2.2 \\
\hline Cutting Voice & Normal & Scream & Normal & Scream & Normal & Noise \\
\hline \multirow{2}{*}{ Workpiece surface topography } & & & & & & \\
& & & & & & \\
\hline Conclusion & Stable & Flutter & Stable & Flutter & Stable & Flutter \\
\hline
\end{tabular}

Table 2 The optimization of cutting parameters table before and after

\begin{tabular}{|c|c|c|c|c|}
\hline \multirow{2}{*}{ The tool parameters } & Spindle speed & Radial cutting depth & Axial cutting depth & The material removal rate \\
\hline & $(\mathbf{r} / \mathbf{m i n})$ & $(\mathbf{m m})$ & $(\mathbf{m m})$ & $\left(\mathrm{cm}^{3} / \mathrm{min}\right)$ \\
\hline \multirow{3}{*}{$\Phi 10 \mathrm{~mm} / 2$ Tooth } & 16000 & 0.1 & 0.81 & 1.3 \\
\hline & 22000 & 4 & 1.5 & 13.2 \\
\hline & 36000 & 4 & 1.5 & 20.4 \\
\hline \multirow{3}{*}{$\Phi 12 \mathrm{~mm} / 2$ Tooth } & 28000 & 0.1 & 1 & 2.8 \\
\hline & 20000 & 5 & 2 & 200.0 \\
\hline & 37000 & 5 & 2 & 370.0 \\
\hline \multirow{3}{*}{ Ф5m/2Tooth } & 25000 & 0.1 & 0.48 & 1.2 \\
\hline & 28000 & 2 & 2 & 112 \\
\hline & 38000 & 2 & 1.5 & 114 \\
\hline \multirow{2}{*}{ Ф5mm/4Tooth } & 20000 & 0.1 & 0.3 & 0.6 \\
\hline & 36000 & 0.2 & 0.5 & 3.6 \\
\hline
\end{tabular}

\section{Conclusions and discussions}

In this paper in order to solve the problem that selects the parameters existing CNC milling 
process, based on Calculation and analysis of the dynamic milling force modeling and flutter stability region, Taking Matlab as the development tool, establishing the regeneration Chatter milling simulation algorithm. The principle and steps of the algorithm are presented in detail. The flutter stability domain graphics of the whole machining system was given out, Because of these theories and algorithms, the selection and optimization of milling parameters is provided theoretical basis. The simulation algorithm is proved to be efficient and accurate by cutting experiment, and in the plant practical application reflect its practical value.

\section{References}

[1] J Gradisek, M Kalveam and K Weinert : Int J Mach Tools Manuf Vol. 401-414(2004), p.44

[2] S Ratchev, S Liu and W Huang, et al: Mater process Technol Vol. 134-138(2004), p.153

[3] S H Ryu, C N Chu: Int J Mach Tools Manuf Vol. 1629-1641(2004), p.44

[4] B A Mascardelli, S S Park and T Freiheit : Journal of Manufacturing Science and Engineering Vol.1-12(2008), p.130

[5] Z Lei, Z Li: International Journal of Machine Tools\&Manufacture Vol. 225-235(2004), p.44

[6] Z Z Li, Z.H Zhang and L Zheng: International Journal of Advanced Manufacturing Technology Vol. 541-552(2004), p.24

[7] U Bravo, O Altuzarra : International Journal of Machine Tools\&Manufacture Vol. 1669-1680(2005), p.45

[8] V Gagnol, B C Bouzgarrou : International Journal of Machine Tools\&Manufacture Vol. 11761186(2007), p.47

[9] E Ozlu, E Budak : Journal of Manufacturing Science and Engineering Vol, 726-732(2007), p.129

[10]Y Altintas, E Shamoto and P Lee, et al: Journal of Manufacturing Science and Engineering Vol. 586-592 (1999), p.121

[11] E Budak, Y Altintas and E.J.A Armarego : Transactions of the ASME Journal of Manufacturing Science and Engineering Vol. 216-224(1996), p. 118 